\title{
Asteroseismology: Lessons From the Past and Prospects for the Future Steven D. Kawaler
}

Dept. of Physics and Astronomy, lowa State University, Ames, IA 50014 USA

\begin{abstract}
Ground-based, and now space-based, studies of a range of stellar families (all represented at this workshop) have, in large part, moved from study of pulsations for their own sake on to information of real value for stellar physics and its applications. This required a combination of improved observations, and open-minded stellar modelling, that continues today. Pulsating compact stars provide a good example of this progress. A flurry of activity from the mid 1980 s to the mid 1990 s, both observational and theoretical, began to realize this potential. A new generation of stellar models, coupled with reanalysis of seismological data and discovery of many new faint pulsators, have recently revitalized this field and may soon provide firm answers to some of the outstanding problems of post-AGB evolution. The discovery and analysis of pulsating sdB stars has followed an accelerated trajectory, enjoying mature theoretical model framework largely in place at the same time as the developing observational base.
\end{abstract}

\section{Compact pulsators: asteroseismology in action}

The story of stellar seismology spans nearly a century if one acknowledges that classical variable stars have revealed fundamental stellar properties such as the mean density of radial pulsators. However, it was the discovery of multimode pulsating stars that precipitated exploration of the details of stellar interior physics and structure. One of the earliest milestones was the 1965 discovery of pulsations in white dwarfs by Arlo Landolt (Landolt 1968). Following this discovery, astronomy soon recognized the importance of these multiperiodic stars. Theoretical exploration led to the discovery of the pulsating DB white dwarfs. Around that time, we were faced with the serendipitous discovery of the pulsating DOZQ1 white dwarfs (pulsating PG 1159-035 stars, or GW Vir stars for the purists). Despite a few fits and starts, we eventually learnt how to analyse them, and now routinely probe interesting features of their interiors: compositional stratification, crystallization, and rotation.

Rather than a comprehensive overview of the voluminous scientific results, I will try to use a few sample results to illustrate how the promise of asteroseismology has been realized, and how our more optimistic views have needed to respond to reality. Twenty years after the discovery of pulsating white dwarfs, Don Winget reviewed the state of play of the seismology of compact objects at IAU Symp. 123 in Aarhus (Winget 1988). Today, twenty years later, that review serves as an interesting touchstone to judge our progress. Here l'll discuss a few examples of the organic growth process in white dwarf seismology.

Nonadiabatic studies and the "thick vs. thin" debate

One of the early achievements of white dwarf seismology was the determination that the ZZ Ceti stars were pulsating in nonradial modes, with partial ionization of hydrogen providing the driving energy and (to some degree) a mode selection mechanism. The history of this discovery is recounted in Winget (1988) and will not be repeated here. This was a very successful theory, whose success can be judged in part by its application to the prediction (and later observational verification) of pulsating DB white dwarf stars by Winget et al. (1982). 
However, consider the following example of the subtleties of nonadiabatic calculations that were not fully appreciated at the time. Stability calculations by Winget and others (e.g. Winget et al. 1982) suggested that only DA white dwarfs with thin $\mathrm{H}$ envelopes $\left(<10^{-8} M_{\odot}\right)$ showed unstable g modes at temperatures corresponding to ZZ Ceti stars. This argument for "thin" envelopes ran counter to evolutionary calculations (e.g. Iben 1984) that showed that hydrogen burning in pre-DA white dwarfs would leave a "thick" layer of $10^{-4} M_{\odot}$. Models by Art Cox and colleagues (e.g. Cox et al. 1987) did not have the same thermal structure in the envelope, and showed instabilities with thick envelopes. After much argument, the "thick" vs. "thin" issue clarified when updated opacities were included in the DA models (i.e. Bradley \& Winget 1994), showing that pulsations could be driven, at the proper temperatures, with "thick" envelopes.

We are now in an era of renewed progress in understanding driving in white dwarfs. This includes the vexing convection-pulsation interaction (e.g. Wu \& Goldreich 2001). Another area of recent, rapid progress is in understanding the driving of pulsations in GW Vir stars. The general picture of driving by $\mathrm{C}$ and O ionization by Starrfield et al. (1983) has been updated recently by several, including Cox (2003), Quirion et al. (2004) and Gautschy et al. (2005).

\section{Mode trapping by composition transition zones}

Through the early 1980s, model studies of the pulsations of white dwarfs concentrated on the driving mechanism of the ZZ Ceti stars. Progress in that area spawned active discussions concerning the thickness of the surface hydrogen layer. Winget et al. (1981) were able to show that certain modes can be concentrated in the surface layers via resonant mode trapping - placing a node in the perturbation eigenfunction near the (steep) composition transition zone separating the hydrogen surface layers from the subsurface helium layer can choke the amplitude of the eigenfunction in the interior. Such modes should therefore be easier to excite (since only the non-degenerate outer parts of the star participate in oscillatory motion). This idea was used in an effort to understand mode selection in white dwarfs - the observed number of frequencies is usually much smaller than the number of modes available to the star.

Mode trapping as a selection mechanism was one way that early work in this field informed the debate about the internal structure of white dwarfs. It is largely an adiabatic property of the modes, and so is at first blush a robust tool. Nonadiabatic studies generally conformed, finding faster linear growth rates in trapped modes. But translation between linear growth rates and observed amplitudes is a difficult process. Without a more complete mode spectrum, or other constraints on global stellar parameters, mode trapping alone is insufficient for providing conclusive inferences about white dwarf structure.

Exploitation of mode trapping to determine subsurface compositional structure had to wait for a different diagnostic - one that relied on the asymptotic modes not generally seen in the ZZ Ceti stars, but familiar to helioseismology. It was the prototype GW Vir star PG 1159-035 that showed the first evidence of a pulsation spectrum that could be analysed with similar asteroseismological tools as were being used on the Sun. An early multi-site observing run on this star enabled Winget et al. (1985) to produce a list of eight frequencies for PG 1159-035 that was free of diurnal aliases and sufficiently resolved. Kawaler $(1986,1988)$ showed that these frequencies formed a partial sequence of modes roughly equally spaced in period (with some missing members), with a common period spacing of about 21 seconds - as expected for an asymptotic g-mode pulsator. For $\ell=1$ modes, a period spacing of 21 seconds was found to be perfectly consistent with models of PG 1159-035 if it had a mass of close to $0.60 M_{\odot}$. This conclusion was based on a statistical treatment of the mode list (see Fig. 1) that showed the pattern was unlikely to be caused by chance.

Figure 1 shows the results of a Kolmogorov-Smirnov test (Kawaler 1988). The K-S statistic reaches a minimum at 21 seconds. While this looks like a convincing result (and was 


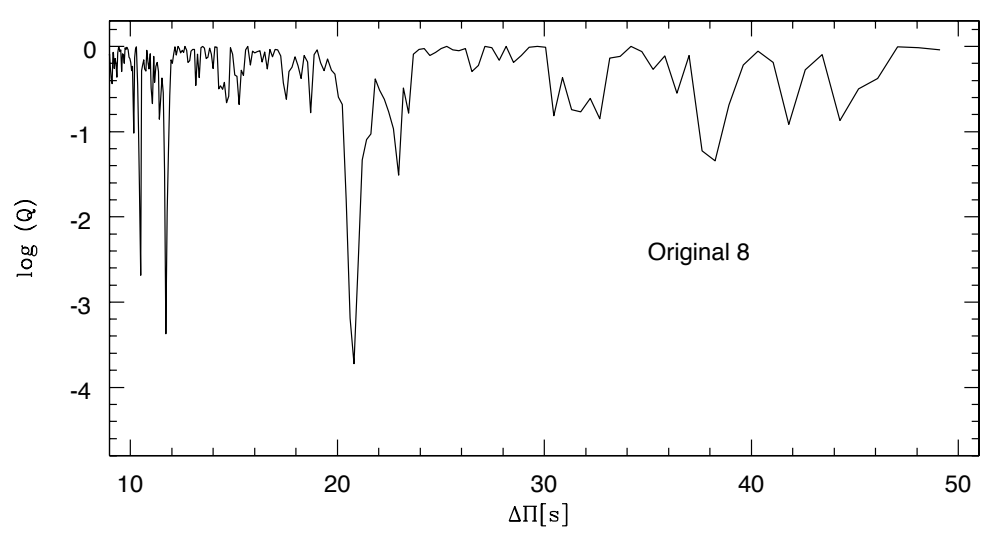

Figure 1: PG 1159-035 period spacing K-S diagnostic using the original eight periods.

taken as such by the author at the time it was first seen) it is difficult to assign an absolute significance level from the K-S statistic Q. Formally, a value of -3 corresponds to a 1 -in-1000 chance of random occurrence, but as can be seen in Fig. 1 two other peaks sit at close to the same significance as the $21 \mathrm{~s}$ signal. A more appropriate and modern approach is to simulate the problem via Monte Carlo techniques, running a large number of trials each with eight random periods selected from the overall range of periods seen in PG 1159-035. Doing a K-S test on each trial, and looking at the relative frequency of the $Q$ statistic for the best spacing for each trial gives a better feel for how significant the result is for PG 1159-035 with the original eight modes.

Figure 2 shows a sample of this kind of Monte Carlo analysis for eight random periods. The significance of the original PG $1159-035$ result is at about the $90 \%$ confidence interval. Another test, the inverse variance test (O'Donoghue 1994), shows similar results. In isolation, the significance of the original period spacing determination seems far from certain. However, coupled with the theoretical expectation of equal spacing in period for high-order $g$ modes, the spacing allowed derivation of an asteroseismic mass for the star. Furthermore, this led to the expectation that if more modes were found, they should fit the pattern.

Within a few years, conclusive observational evidence followed from a global, coordinated observing effort - the Whole Earth Telescope (Nather et al. 1990). The WET observations of PG 1159-035 (Winget et al. 1991) were of much higher quality and density than any single-site efforts. They fully resolved the multiplets in PG 1159-035 and uncovered a nearly complete sequence of $\ell=1$ overtones along with several $\ell=2$ modes. With those new modes added, the K-S statistic $(\log Q)$ for the best-fit spacing of 21 seconds fell to -31.0 , with a confidence interval of much better than $99.99 \%$.

The complete set of modes provides a deeper seismic inference for PG 1159-035. As shown by Winget et al. (1991) and Kawaler \& Bradley (1994) for GW Vir stars (and for DB stars by Bradley et al. 1993), departures from uniform spacing in period are characteristic of trapped modes. They were able to compare the observed departures with models to make an asteroseismological determination of the thickness of the surface $\mathrm{He}+\mathrm{C} / \mathrm{O}$ layer in PG 1159-035.

Application of this adiabatic mode-trapping determination to DA models soon followed with the work of Brassard et al. (1992) and Kawaler and Weiss (1991), following up on analytic work by $\mathrm{C}$. Hansen (unpublished). These basic tools remain central ideas in white dwarf seismology today, and have led to determinations of white dwarf masses and other 


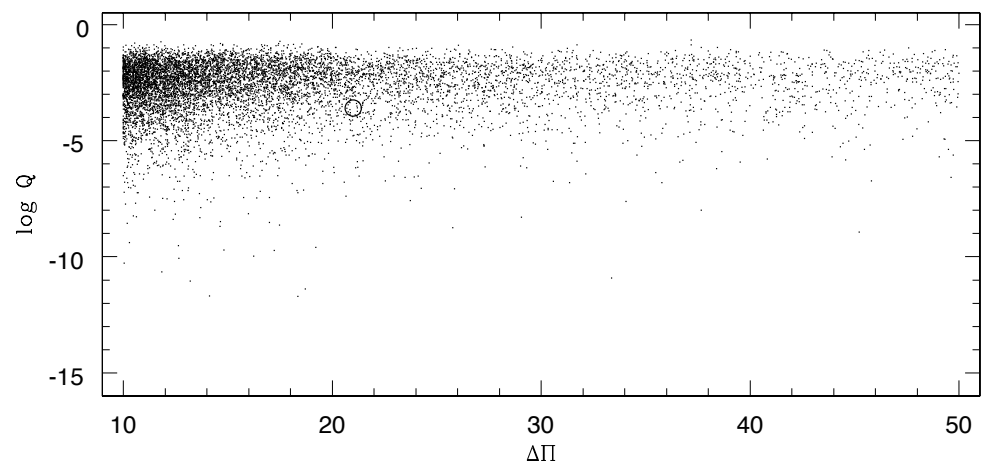

Figure 2: Monte Carlo simulation of 10000 trials of eight randomly chosen periods. Each point shows log $\mathrm{Q}$ for the best period spacing. The original PG 1159-035 spacing (circle) shows a constant period spacing at about the $90 \%$ confidence level.

Table 1: Rotational splitting deviations in pulsating white dwarfs

\begin{tabular}{lccc} 
& $\delta \nu / \nu$ & \multicolumn{2}{c}{ variation $(\%)$} \\
Star & {$[\%]$} & within multiplets & between multiplets \\
\hline NGC 1501 & 1.51 & 2.0 & 6.0 \\
PG 2131 & 1.07 & 13.2 & 3.1 \\
RX J2117 & 0.504 & $(3.4)$ & 20.0 \\
GD 358 & 0.340 & 14.5 & 3.9 \\
PG 1159 $\ell=1$ & 0.222 & 4.9 & 1.3 \\
HL Tau 76 & 0.173 & 10.2 & 3.5 \\
GD 165 & 0.057 & 7.0 & 9.0
\end{tabular}

properties for a growing number of stars... including a probable detection of a crystalline core in the massive ZZ Ceti star BPM 37093 (Montgomery \& Winget 1999, Metcalfe et al. 2004, Corsico et al. 2005, Kanaan et al. 2005).

\section{Faith and rotational splitting}

Studies of rotational splittings in pulsating stars rely on several fundamental "truths." First is that rotational splitting produces multiplets with equal splittings. When all $\mathrm{m}$ modes are present, $2 \ell+1$ peaks appear, equally spaced in frequency by a multiple of the rotation frequency. For uniform rotation with depth in a star, all multiplets show the same splitting.

In practice, though, we find that all of these assumed truths are not fully realized. Table 1 illustrates this with published results for a number of white dwarf pulsators. In most cases, the departures from uniformity are small but still much larger than the observational uncertainty, ranging from a few percent up to 20 percent variation from the mean spacing. There is no clear trend for fractional asymmetry to increase as the ratio of rotation rate to pulsation frequency increases (as one might expect from basic theory).

Other physics can play a role. Magnetic field effects which can shift the $m=0$ peak within a multiplet and produce non-uniform spacing (Jones et al. 1989, Winget et al. 1991). Some members of multiplets may not be visible (or even excited). Uniform rotation with depth does not necessarily produce equally spaced multiplets across several overtones because of mode 
trapping effects (Kawaler et al. 1999, Goupil et al. 2000). And differential rotation within stars could produce quite varied spacings (i.e. Goupil et al. 1996, Kawaler and Hostler 2005). Also, as rotationally split multiplets are nearly in 1:1 resonance, nonlinear effects can play a role in perturbing the observed frequencies.

Given all of the above, we should not be surprised that the observed frequency spacings in multiplets in white dwarfs show significant departures from the expected truths. Interpretation of these asymmetries, however, needs to be approached with some caution. Initially, the changing splittings in GD 358 were interpreted as direct evidence for differential rotation (i.e. Winget et al. 1994), but further analysis by Kawaler et al. (1999) revealed the influence of mode trapping on the splittings. And in some sdB stars, relatively large, equally split multiplets appear (i.e. PG 1605, Kawaler 1999 and PG 0014, Vuckovic et al. 2006) that are not easily reconciled with limits on surface rotation velocities.

\section{The pulsating sdB stars}

The 40 year history of pulsating white dwarf studies has been repeated, on a much compressed time scale and with some interesting complications, in the development of sdB seismology. This field, ably reviewed by Stephane Charpinet at this workshop, was enabled in part by the experience gained by asteroseismologists in developing white dwarf seismology.

In the case of the sdB stars, as in the white dwarfs, the discovery of the pulsator, EC 14026, was through a sequence of serendipitous events. The account of their 1995 discovery by Kilkenny et al. (1997) ranks, in my opinion, as one of the most engaging tales to appear in the refereed astronomical literature. It should be required reading for all graduate students embarking on a research career, as Kilkenny et al. (1997) stress that one must always keep an open mind when dealing with astronomical data. Their ultimate words bear quoting here as it has turned out to apply at so many stages in asteroseismology: "Despite a programme of careful observation, serendipity appears to have a major role to play in research and we are forcibly reminded that if we only look for what we expect to find, we might well miss exciting new discoveries."

Simultaneously, and without knowing that the South African group had found these stars, the Montreal group had done some exploratory calculation of the pulsational instability of sdB stars, and predicted that some should indeed pulsate (Charpinet et al. 1996). When the discovery of the EC 14026 stars was announced, it took only minor modification of their models to match the basic parameters - stellar and pulsational - of the new variable stars (Charpinet et al. 1997). For further details, see Stephane's review in these proceedings.

Only 10 short years later, models of these stars correspond even more closely to the observations - and demonstrate the potential of asteroseismology to probe important interior processes such as radiative levitation and winds. It took nearly twice as long to reach a similar level of detail with the white dwarfs.

\section{Conclusions}

In conclusion, we live in a very special time - for most of us of the "Breger-and-students" generation, the field of asteroseismology was born, went through a stormy adolescence, and is reaching maturity. The big brother (in terms of age) of the asteroseismology family, the pulsating white dwarfs, have already left the nest and become a mature area of detailed study, joined by the $\delta$ Scuti stars. In Don Winget's review (Winget 1988) many of the expectations were met, other promises still await fulfilment, and entirely unexpected developments have, as expected, been made. Other younger siblings, such as the sdB stars, and SPB stars and solar-like pulsators, are still in the growth phase, and it will be a lot of fun to watch those stars, and other kinds yet to be discovered, teach us about stellar evolution from the inside out in the coming decades. 
Acknowledgments. The author thanks Gerald Handler and the other organizers of this celebration for their invitation, and for some travel support. Additional support came from NASA Grant NNG05-GG20G.

\section{References}

Bradley P. A., Winget D. E., Wood M. A., 1993, ApJ, 406, 661

Bradley P. A., Winget D. E., 1994, ApJ, 421, 236

Brassard P., Fontaine G., Wesemael F., Hansen C. J., 1992, ApJS, 80, 369

Charpinet S., Fontaine G., Brassard P., Dorman B., 1996, ApJL, 471, L103

Charpinet S., Fontaine G., Brassard P., et al., 1997, ApJL, 483, L123

Corsico A. H., Althaus L. G., Montgomery M. H., Garcia-Berro E., Isern J., 2005, A\&A, 429, 227

Cox A. N., Kidman R. B., Starrfield S. G., Pesnell W. D., 1987, ApJ, 317, 303

Cox A. N., 2003, ApJ, 585, 975

Iben I. Jr., 1984, ApJ, 277, 333

Gautschy A., Althaus L. G., Saio H., 2005, A\&A, 438, 1013

Goupil M.-J., Dziembowski W. A., Goode P. R., Michel E., 1996, A\&A, 305, 487

Goupil M.-J., Dziembowski W. A., Pamyatnykh A. A., Talon S., 2000, in Breger M., Montgomery M. H., eds, ASP Conf. Ser. Vol. 210, Delta Scuti and Related Stars. Astron. Soc. Pac., San Francisco, p. 267

Jones P., Hansen C. J., Pesnell W. D., Kawaler S. D., 1989, ApJ, 336, 403

Kanaan A., Nitta A., Winget D. E., et al., 2005, A\&A, 432, 219

Kawaler S. D., 1986, Ph.D. dissertation, University of Texas

Kawaler S. D., 1988, in Christensen-Dalsgaard J., Frandsen S., eds, IAU Symp. 123: Advances in Helioand Asteroseismology, Reidel, Dordrecht, p. 329

Kawaler S. D., Weiss P., 1990, in Osaki Y., Shibahashi H., eds, Progress of Seismology of the Sun and Stars. Lecture Notes in Physics, Vol. 367, Springer Verlag, Heidelberg, p. 431

Kawaler S. D., Bradley P. A., 1994, ApJ, 427, 415

Kawaler S. D., 1999, in Solheim J.-E., Meistas E., eds, ASP Conf. Ser. Vol. 169, 11th European Workshop on White Dwarfs. Astron. Soc. Pac., San Francisco, p. 158

Kawaler S. D., Sekii T., Gough D. O., 1999, ApJ, 516, 349

Kawaler S. D., Hostler S. R., 2005, ApJ, 621, 432

Landolt A. U., 1968, ApJ, 153, 151

Kilkenny D., Koen C., O'Donoghue D., Stobie R., 1997, MNRAS, 285, 640

Metcalfe T. S., Montgomery M. H., Kanaan A., 2004, ApJL, 605, L133

Montgomery M. H., Winget D. E., 1999, ApJ, 526, 976

O'Donoghue D., 1994, MNRAS, 270, 222

Quirion P.-O., Fontaine G., Brassard P., 2004, ApJ, 610, 436

Vučković M., Kawaler S. D., O'Toole S., et al., 2006, ApJ, 646, 1230

Winget D. E., Van Horn H. M., Tassoul M., et al., 1982, ApJL, 252, L65

Winget D. E., Van Horn H. M., Hansen C. J., 1981, ApJL, 245, L33

Winget D. E. 1988, in Christensen-Dalsgaard J., Frandsen S., eds, IAU Symp. 123: Advances in Helioand Asteroseismology, Reidel, Dordrecht, p. 305

Winget D. E., Nather R. E., Clemens J. C., et al., 1991, ApJ, 378, 326

Winget D. E., Nather R. E., Clemens J. C., et al., 1994, ApJ, 430, 839

Wu Y., Goldreich P. 2001, ApJ, 546, 469 


\section{DISCUSSION}

Roxburgh: You started off by asking what are the big questions and then stated your own interests. I am sitting on a committee that is dominated by cosmologists and astrobiologists. What part of what you do is of interest to these two factions?

Kawaler: What is immediately obvious is white dwarf chronology by finding the ask of the disk of our Galaxy. A rather unexpected outcome would be models of planet formation using stellar evolution codes but also watching the planets grow.

Montgomery: I would just like to state that in the past we haven't used the combination frequencies very much but I am just in the process of doing that, generating nonlinear models of light curves. There is a lot of promise in that, such as constraining models of convection in white dwarf envelopes. I am just plugging my poster here.

Dziembowski: What is the magic about 1.52 ?

Kawaler: There were some stars whose names I don't remember now, that had frequencies at multiples of $1.52,2.52$ etc. or $1.48,2.48$ etc. of the dominating mode frequency. So it was about half way in between harmonics, offset by some constant $\epsilon$.

Dziembowski: And what was the explanation of this?

Kawaler: There were peaks, but they just turned out to be modes that happened to be close. It turned out that $2 / 100$ of that frequency interval was just not that close.

Moskalik and Handler: These stars were PG 1351+489 and GD 154.

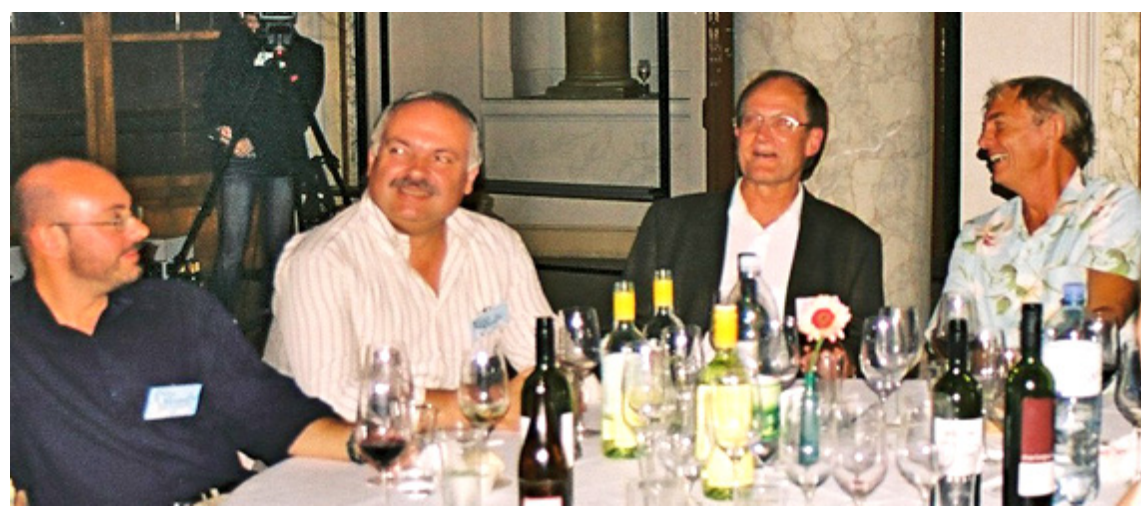

Runa Briguglio, Ennio Poretti, Michel Breger and Jean-Claude Valtier. 


\section{Ground-based asteroseismology}

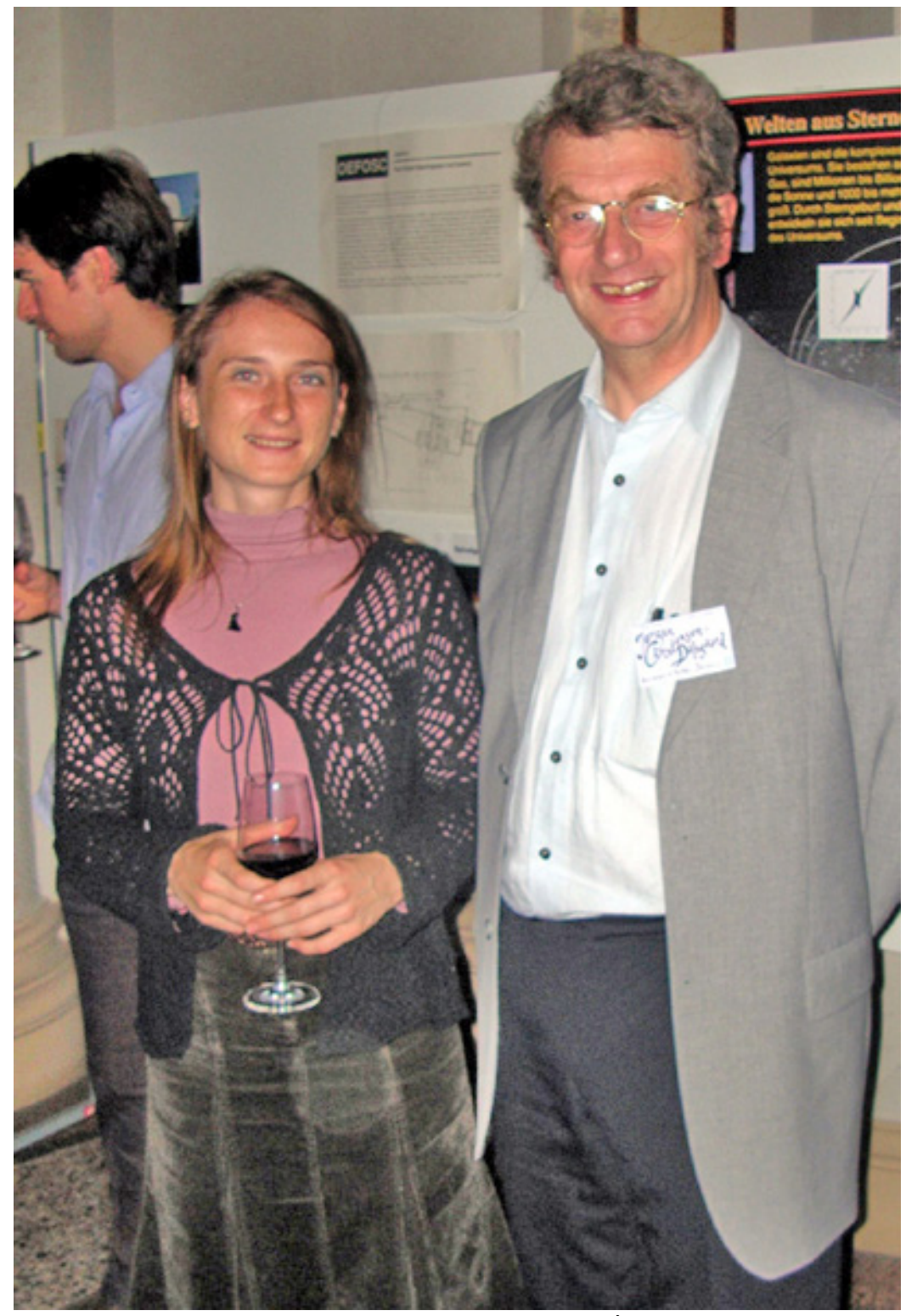

Two ground-based astronomers: Joanna Molenda-Żakowicz and Jørgen Christensen-Dalsgaard. 